\title{
Optimization of Retardance for a Complete Stokes Polarimeter
}

\author{
D. S. Sabatke, M. R. Descour, and E. L. Dereniak \\ Optical Sciences Center, University of Arizona, Tucson, Arizona 85721 \\ W. S. Sweatt, S. A. Kemme, and G. S. Phipps \\ Sandia National Laboratories, Albuquerque, New Mexico 87185
}

\section{MERED \\ JAN 242090}

\begin{abstract}
We present two figures of merit based on singular value decomposition which can be used to assess the noise immuntyte
a complete Stokes polarimeter. These are used to optimize a polarimeter consisting of a rotatable retarder and fixed a complete Stokes polarimeter. These are used to optimize a polarimeter consisting of a rotatable retarder and fixed found to be optimal when four measurements are used. Use of this retardance affords a factor of 1.5 improvement in signal-to-noise ratio over systems employing a quarter wave plate. A geometric means of visualizing the optimization process is discussed, and the advantages of the use of additional measurements are investigated. No advantage of using retarder orientation angles spaced uniformly through $360^{\circ}$ is found over repeated measurements made at the four angles given previously.
\end{abstract}

Measurements of the polarization state of light are important in many fields, including atomic spectroscopy, optical scattering, and remote sensing. The polarization state of light is often expressed in terms of the Stokes vector, a 4-element vector whose components represent respectively the total power in a beam, a preference for horizontal vs. vertical polarization, a preference for $45^{\circ}$ vs. $135^{\circ}$ polarization, and a preference for right- vs. left-handed circular polarization. ${ }^{2}$ A complete Stokes polarimeter is an instrument which measures the Stokes vector of light. In this article we present two figures of merit for assessing the noise immunity of a Stokes polarimeter, and employ them in optimizing a system which uses a rotatable retarder and fixed linear polarizer as its polarization-analyzing optics. We also discuss an interesting means of visualizing the optimization process, and investigate the impact of the number of measurements made with the polarimeter on its performance.

We employ a general model of a polarimeter as an instrument which acquires data by measuring the power remaining in a beam after passing through a system of polarization optics described by known Mueller matrices. If a total of $N$ power measurements $p_{k}, k \in\{0,1, \ldots N-1\}$ are made, each through a system of Mueller matrix $M_{k}$, the measurements are related to the Stokes vector $\bar{S}$ of the incident beam in the absence of noise via

$$
\bar{P}=\mathbf{W} \bar{S} \text {. }
$$

Here $\vec{P}$ is an $N$-element column vector containing the $p_{k}$ and $W$ is the $\mathrm{N} \times 4$ measurement matrix ${ }^{3}$. The $\mathrm{k}^{\text {th }}$ row of $W$ is computed as $\bar{A}^{T} \mathbf{M}_{k}$, where $\bar{A}$ is a 4-element column vector describing the polarization-sensitive responsivity of the detector.

The measurement matrix $W$ in general might not be invertible. (If $N \neq 4$ it will not even be square.)

Therefore the pseudoinverse is used to reconstruct an estimate $\hat{S}$ of the incident Stokes vector as

$$
\hat{S}=\mathbf{W}^{+} \bar{P} \text {. }
$$

Here a superscripted + represents the pseudoinverse operation. The pseudoinverse is identical to the matrix inverse when the inverse exists, and results in a minimum-norm, least-squares reconstruction otherwise. ${ }^{4}$ In order to describe a real system in which noise is present, Eq. 1 should be replaced by

$$
\bar{P}=\mathrm{W} \bar{S}+\bar{n}
$$

where $\vec{n}$ is an $N$-element noise vector whose elements may be considered random variables. Substitution into Eq. 2 yields a term

$$
\delta \hat{S}=\mathrm{W}^{+} \bar{n}
$$

which represents the error due to noise in the Stokes vector measurement. A number of figures of merit have been used to quantify the effects of this term, some of which are restricted in applicability to the case of 4 measurements so that $W$ is square. We discuss briefly two figures of merit which can be applied for arbitrary $N$ and have simple representations in the context of singular value decomposition.

- We denote the first figure of merit $\mathrm{RAD}$ (for reciprocal absolute determinant), and define it by 


\section{DISCLAIMER}

This report was prepared as an account of work sponsored by an agency of the United States Government. Neither the United States Government nor any agency thereof, nor any of their employees, make any warranty, express or implied, or assumes any legal liability or responsibility for the accuracy, completeness, or usefulness of any. information, apparatus, product, or process disclosed, or represents that its use would not infringe privately owned rights. Reference herein to any specific commercial product, process, or service by trade name, trademark, manufacturer, or otherwise does not necessarily constitute or imply its endorsement, recommendation, or favoring by the United States Government or any agency thereof. The views and opinions of authors expressed herein do not necessarily state or reflect those of the United States Government or any agency thereof. 


\section{DISCLAIMER}

Portions of this document may be illegible in electronic image products. Images are produced from the best available original document. 


$$
R A D=\prod_{j=0}^{R-1} \frac{1}{\mu_{j}}
$$

Here $R$ is the rank of $W$ and the $\mu_{j}$ are its singular values. The product runs over only the non-zero singular values (of which there are four for a complete polarimeter). In the case $W$ is square and invertible, the RAD is equal to the reciprocal of the absolute value of its determinant. It provides a means of visualizing the optimization process in geometric terms which will be discussed shortly. For $N>4, \mathrm{RAD}$ is a meaningful generalization of figures of merit based on the determinant used by other authors. 5

The second figure of merit is derived by considering the variances of the components of the Stokes vector estimate. Let the variance of the noise in the $k^{\text {th }}$ measurement (i.e. in the $k^{\text {th }}$ component of $\bar{n}$ ) be denoted $v n_{k}$. It is reasonable to assume that the noise between measurements is uncorrelated, in which case it follows from Eq. 4 that the variance $v_{j}$ in the $j$ th component of the estimated Stokes vector is

$$
v_{j}=\sum_{k=0}^{N-1}\left(\mathbf{W}^{+}\right)_{j, k}^{2} v n_{k}
$$

If all measurements have the same variance, it can be factored out yielding a variance amplification

$$
\frac{v_{j}}{v n}=\sum_{k=0}^{N-1}\left(\mathbf{W}^{+}\right)_{j, k}^{2}
$$

for the $j^{\text {th }}$ Stokes component. The assumption of equal variances is justified in at least two situations: 1.) when the noise is (signal-independent) detector noise whose statistics don't change between measurements, and 2.) when the polarization optics consist of a rotatable retarder followed by a fixed polarizer and the $S_{0}$ component of the incident Stokes vector is much larger than any of the other components (as is expected for naturally illuminated scenes), so that the noise in all measurements is due to signal-dependent noise in the detection of $S_{0}$.

If a particular Stokes vector component were of interest, the corresponding variance amplification factor could be used as a figure of merit. When all Stokes vector components are equally of interest, it is reasonable to form a figure of merit by summing all four factors with equal weights. We refer to this figure of merit as EWV (for equally weighted variance).

$$
E W V=\sum_{j=0}^{3} \sum_{k=0}^{N-1}\left(\mathbf{W}^{+}\right)_{j, k}^{2}=\operatorname{Tr}\left\{\left(\mathbf{W}^{+}\right)^{\mathrm{T}} \mathbf{W}^{+}\right\}
$$

This is simply the sum of squares of all the elements of $W^{+}$, which we note can be written as the trace of the product of $W^{+}$and its transpose. The latter form allows EWV to be expressed in terms of the singular values of $W$ as

$$
E W V=\sum_{j=0}^{R-1} \frac{1}{\mu_{j}^{2}}
$$

Since both RAD and EWV feature singular values of $W$ in the denominator, they exhibit similar behavior. In particular, they tend to infinity as $W$ approaches singularity. Noting that the pseudoinverse $W^{+}$in the error term given by Equation 4 entails division by the singular values, it is seen that these figures of merit should be minimized to promote noise immunity.

These figures of merit have been applied to the optimization of a polarimeter featuring a rotatable retarder followed by a fixed linear polarizer and polarization-insensitive detector. This is a common configuration, and in the past has often been implemented with a quarter wave plate. However a quarter wave retardance is not the optimal value, when judged by the figures of merit RAD and EWV. Denoting the retardance $\delta$ and letting the retarder be rotated (about the optical axis) to an angle $\theta_{k}$ relative to the transmissive axis of the polarizer for the $\mathrm{k}^{\text {th }}$ measurement, the rows of the measurement matrix $W$ are proportional to

$$
\text { (1 } \left.\cos ^{2}\left(2 \theta_{k}\right)+\sin \delta \sin ^{2}\left(2 \theta_{k}\right) \quad \sin ^{2}(\delta / 2) \sin \left(4 \theta_{k}\right)-\sin \delta \sin \left(2 \theta_{k}\right)\right) \text {. }
$$

We carried out a numerical minimization in Mathematica ${ }^{8}$ of the EWV figure of merit for the case of $N=4$ measurements with respect to five variables ( $\delta$ and the four retarder orientation angles). We assumed a polarization insensitive detector. A polarization insensitive detector is described by setting

$$
\bar{A}^{T}=\left(\begin{array}{llll}
1 & 0 & 0 & 0
\end{array}\right) \text {. }
$$


A favorable configuration was found with retardance of $132^{\circ}$ and retarder angles of $\pm 51.7^{\circ}$ and $\pm 15.1^{\circ}$. The minimum is fairly broad. The EWV figure of merit increases from its minimum value of 10.0 to 10.1 with variations in retarder orientation angles on the order of $\pm 2.5^{\circ}$ or variation in retardance of $\pm 4^{\circ}$ about the optimum values. As will be discribed next, this configuration is also optimal with respect to RAD.

Ambirajan and Look have presented an insightful way of visualizing the optimization process. Note that the three-dimensional coordinate given by the last three components of the matrix row in Eq. 10 lies on a sphere (which can be identified as the Poincare sphere ${ }^{10}$ ) of unit radius. The four rows of the measurement matrix thus specify the four vertices of a tetrahedron inscribed in the sphere. Ambirajan and Look point out that the volume of this tetrahedron is proportional to the determinant of the measurement matrix, and that the volume is maximized (RAD is minimized) when the vertices are positioned so as to form a regular tetrahedron. For a given retardance, the locus of coordinates is a curve on the sphere as shown in Figure 1. A configuration which is optimal with respect to RAD is then represented by four points selected on a single curve which form a regular tetrahedron.

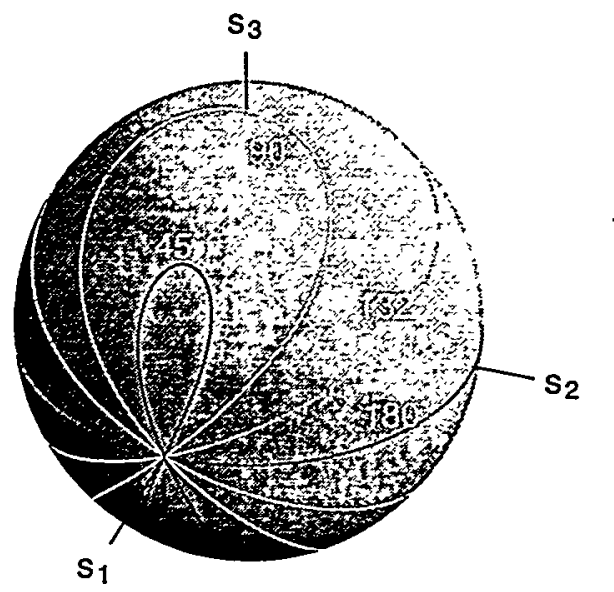

Figure 1. Each trajectory is the locus of points on the Poincare sphere which can be selected by choice of retarder orientation $\theta$ for a given retardance.

Figure 2, part a illustrates an optimal configuration with the curve for a $132^{\circ}$ retardance and one possible inscribed regular tetrahedron. The curve for a quarter wave plate $\left(90^{\circ}\right.$ retardance) is also shown. Other optimal configurations are obtained by reflections of the tetrahedron in the $S_{1}, S_{2}$-plane or the $S_{1}, S_{3}$-plane, which corresponds to addition of the same integer multiple of $90^{\circ}$ to each of the retarder orientation angles. Figure 2, part $b$ shows the tetrahedron for such a case. $90^{\circ}$ increments to the retarder orientations corresponds simply to an exchange of fast and slow axes. Similarly a retardance of $228^{\circ}$ allows optimum configurations. $228^{\circ}$ is equivalent to $-132^{\circ}$, and reversing the sign of retardance amounts to an exchange of fast and slow axes.

\section{$\therefore$}




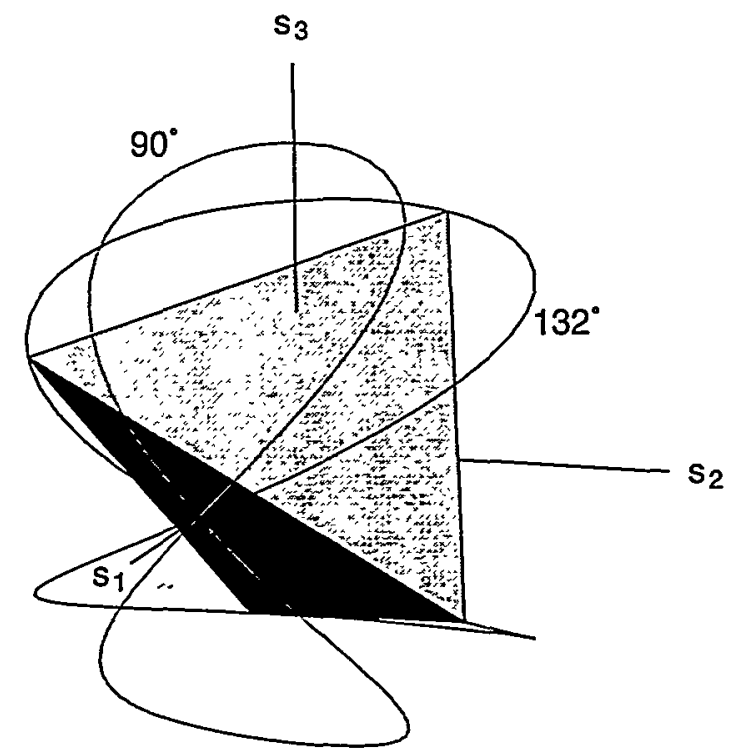

(a)

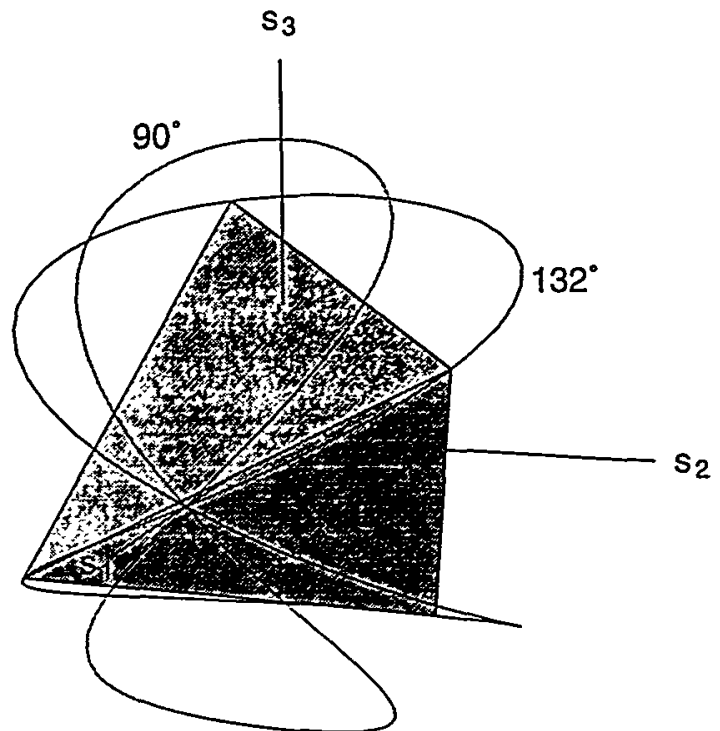

(b)

Figure 2. The curves for retardances of $90^{\circ}$ and $132^{\circ}$, showing an inscribed regular tetrahedron in the curve for $132^{\circ}$. In part a the curve for retarder angles of $\pm 51.7^{\circ}$ and $\pm 15.1^{\circ}$ are shown. Part $b$ shows the tetrahedron obtained when $90^{\circ}$ is added to each of these angles.

sis

The process of selecting four vertex locations which create a regular tetrahedron can be viewed qualitatively as trying to spread the vertices as far apart as possible on the sphere's surface. We can arrive at this interpretation by another method. The (in this case unattainable) goal of ideal conditioning is for the measurement matrix to be unitary, in which case its rows would be mutually orthogonal. Considering the 4-dimensional inner product of two rows given by Equation 10, it is seen that the first components contribute unity. To make the inner product zero, it is necessary for the remaining three components to contribute -1 . That is, the 3-dimensional inner product of the position vectors of the two points on the sphere should be -1 , or the points should be diametrically opposed across the sphere. Clearly it is impossible to have four points on a sphere all mutually opposed, so one must settle for the approximation of spreading them apart as far as possible on the sphere's surface.

The preceding analysis dealt only with the case of $N=4$ measurements. In order to reduce noise, it is common to use additional measurements. The question arises of whether there is any advantage to making these at 
different retarder orientation angles, or if repeated measurements should be made at the angles already found to be optimal for four measurements. We are interested in particular in a comparison with the well-known scheme in which $N$ measurements are taken at angles spaced uniformly over $360^{\circ}$, and data reduction employs a discrete Fourier transform. ${ }^{11}$ To investigate this issue, the EWV figure of merit was calculated as a function of $N$ for three approaches: 1.) measurements made at $N$ angles spaced uniformly through $360^{\circ}$ with a retardance of $90^{\circ}, 2$.) as in case 1 but with a retardance of $132^{\circ}$, and 3.) repeated measurements made at the four optimal angles found earlier with a retardance of $132^{\circ}$. The results are shown in Figure 3.

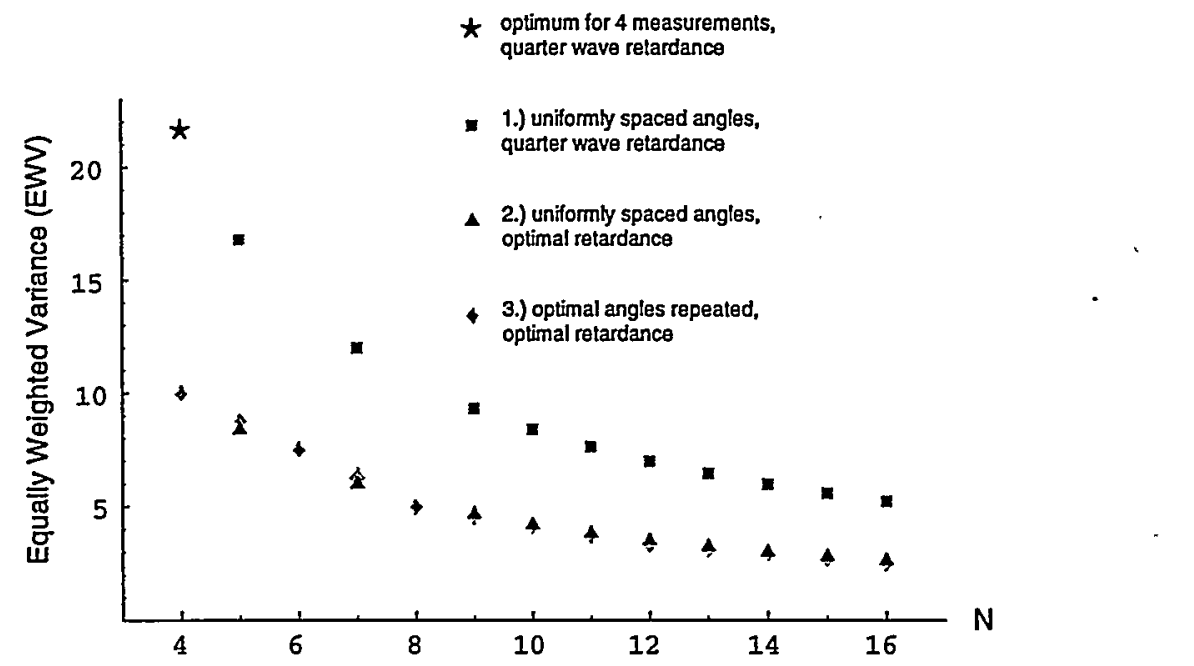

Figure 3. Comparison of multiple measurement techniques, showing values of the EWV figure of merit as a function of the number $N$ of measurements for the three cases described in the text. Missing points at $N=4,6$, and 8 in the data for cases 1 and 2 are due to singularities in the measurement matrices. A single data point (represented by the $\star$ ) is also shown for a four measurement procedure using a quarter wave plate.

All three data sets in Figure 3 display the1/N trend expected in a measure of variance. The cases employing a retardance of $132^{\circ}$ exhibit superior noise immunity to that employing $90^{\circ}$ retardance. There is no appreciable difference as far as EWV is concerned in the perfromance in cases 2 and 3 (equally spaced vs. repeated optimal angles) as long as the values of $N$ which lead to singular matrices in case 2 are avoided. It can be concluded then that the practical difficulty and time expenditure of aligning the retarder at many different angles are unnecessary. Equally good performance is expected with repeated measurements at the four optimal angles. It should be noted that the measurements taken at each of these four angles can be averaged before data reduction, and the data reduction step treated as for a 4-element measurement vector $\bar{P}$ and $4 \times 4$ matrix $W$, thus avoiding the computational costs of larger $N \times 4$ matrices.

Figure 3 also indicates (by the $\star$ symbol) the performance of a system using a quarter wave plate with orientation angles of $-90,-45,30$, and $60^{\circ}$. These angles were recommended as optimal for a quarter wave retardance in ref. 5. The square root of the ratio of the EWV figures of merit for this configuration and the optimal four measurement configuration we have reported yields an average signal-to-noise ratio improvement afforded by our configuration of 1.5 .

In summary, two figures of merit have been presented for the assesment of polarimeter designs without necessitating an assumption on the number of measurements to be used. These have been used to study a common polarimeter architecture consisting of a rotatable retarder followed by a fixed polarizer. The EWV figure of merit was used to optimize the system with respect to retarder orientations as well as retardance for the case of four measurements, indicating that a $132^{\circ}$ retarder is preferable to a quarter wave plate. Use of this retardance allows a factor of 1.5 improvement in signal-to-noise ratio over systems employing a quarter wave retardance. The RAD figure of merit provided a means of visualization of the optimization process and confirmed the validity of the design. The EWV figure of merit can be conveniently interpreted in terms of the system's noise variance, and has 
been used to investigate the effects of using more than four measurements. No appreciable advantage in using angles other than those found in the four angle optimization was observed.

Sandia is a multiprogram laboratory operated by Sandia Corporation, a Lockheed Martin Company; for the United States Department of Energy under contract DE-AC04-94AL895000.

${ }^{1}$ Polarization importance refs.

${ }^{2}$ Azzam and Bashara. Stokes vectors.

${ }^{3}$ R.A Chipman Handbook of Optics $\$ 22.20$.

${ }^{4}$ pseudoinverse reference.

${ }^{5}$ A. Ambirajan and D.C. Look, Opt. Eng. 34, 1651 (1995).

${ }^{6} \mathrm{El}$-Saba, optimization of 4 detector polarimeter, optimization of DOE polarimeter:

${ }^{7}$ Existing instruments with quarter wave retarders.

${ }^{8}$ Mathematica

${ }^{9}$ A. Ambirajan and D.C. Look, Opt. Eng. 34, 1656 (1995).

${ }^{10}$ Born and Wölf, Poincaré sphere

${ }^{11}$ H. G. Berry, G. Gabrielse, and A. E. Livingston, Appl. Opt. 16, 3200 (1977). 